ТЕХНИКО-ЭКОНОМИЧЕСКАЯ ЭФФЕКТИВНОСТЬ ВНЕДРЕНИЯ ДИАГНОСТИКИ КАБЕЛЕЙ НА ПРЕДПРИЯТИЯХ НЕФТЕГАЗОВОЙ ОТРАСЛИ

\title{
TECHNICAL AND ECONOMIC EFFICIENCY OF INTRODUCING DIAGNOSTICS OF CABLES AT OIL AND GAS INDUSTRIES
}

\author{
Новикова Фрейре Шавиер Ж. да К.
}

Уфимский государственный нефтяной технический университет,

\author{
г. Уфа, Российская Федерация \\ G. da K. Novikova Freyre Shavier
}

Ufa State Petroleum Technological University, Ufa, Russian Federation e-mail: shavier_93@mail.ru

Аннотация. Проблема развития нефтегазовой отрасли в Российской Федерации в настоящее время особенно актуальна, так как она непосредственно переплетена со всеми сферами народного хозяйства и играет огромную роль не только в экономике, но и во внешней политике страны. Поэтому в данную отрасль экономики ежегодно вкладываются крупные средства, и правильно их распределить в нужное направление, подобрать более рациональный вариант - вопрос большой значимости. Для того, чтобы вложить больше в техническое развитие нефтегазовой отрасли, нужно минимизировать чрезвычайные расходы.

Одними из таких расходов являются отказы и неисправности кабельных линий на предприятиях нефтегазовой отрасли, приводящие к пожарам, нарушениям технологического процесса и авариям, соответственно влекущими за собой незапланированные расходы, вызывающими экономический ущерб. В большинстве случаев отказ 
кабелей происходит из-за пробоя изоляции во время испытаний повышенным выпрямленным напряжением. Данное испытание классифицируют как косвенно разрушающий метод. Поэтому предлагается внедрение диагностики кабелей с использованием неразрушающего контроля, что позволит в скором времени отказаться от периодических испытаний разрушающими методами. Приведен расчет техникоэкономической эффективности от внедрения предлагаемого метода диагностики на предприятиях нефтегазовой отрасли.

Abstract. The problem of the oil and gas industry development in Russian Federation is currently particularly relevant, as it is directly intertwined with absolutely all spheres of the national economy, and plays a huge role not only in the economy, but also in the foreign policy of the country. Therefore, large funds are invested annually in this sector of the economy, and to properly distribute them in the right direction, to choose a more rational option is a matter of great importance. In order to invest more in the oil and gas industry technical development, it is necessary to minimize extraordinary expenses. One of these costs are the failures and malfunctions of cable lines at the oil and gas enterprises, leading to fires, process violations and accidents, respectively, resulting in unplanned costs - economic damage. In most cases, cable failure occurs due to insulation breakdown during tests with increased rectified voltage. This test is classified as an indirect destructive method. Therefore, it is proposed to introduce cable diagnostics using non-destructive testing, which will soon give up the periodic testing of destructive methods. The calculation of technical and economic efficiency from the implementation of the proposed diagnostic method at the enterprises of the oil and gas industry is given.

Ключевые слова: нефтегазовая отрасль; эффективность; кабель; диагностика; неразрушающий контроль; обеспечение безопасности; испытание; чрезвычайные расходы; капитальные вложения; экономические показатели 
Key words: oil and gas industry; efficiency; cable; diagnostics; nondestructive testing; safety assurance; testing; emergency expenses; capital investments; economic indicators

В настоящее время развитие и роль нефтегазовой отрасли в Российской Федерации особенно актуально и имеет важнейшее значение для экономики и внешней политики страны. Россия считается одним из ключевых поставщиков углеводородного сырья на мировые рынки. На данные ресурсы спрос всегда превышает предложение. Следовательно, характер нефтегазовой отрасли - развивающийся, поэтому в данную отрасль экономики ежегодно вкладываются крупные средства, правильно их распределить, подобрать более рациональный вариант - вопрос большой значимости [1].

Главным признаком технического развития любой отрасли является уровень производительности труда. Производительность труда выше, соответственно, и продукции на единицу времени создается больше. А для того, чтобы вложить больше в техническое развитие нефтегазовой отрасли, нужно минимизировать чрезвычайные расходы [2]. Одними из таких расходов являются отказы и неисправности электрооборудования на промышленных предприятиях, приводящие к пожарам, нарушениям технологического процесса и авариям, следовательно, влекущие за собой незапланированные расходы - экономический ущерб.

Основная часть аварийности на предприятиях нефтегазовой отрасли приходится на кабельные линии, это примерно $62 \%$ от всего электрооборудования за период 2015-2018 гг. (рисунок 1). 


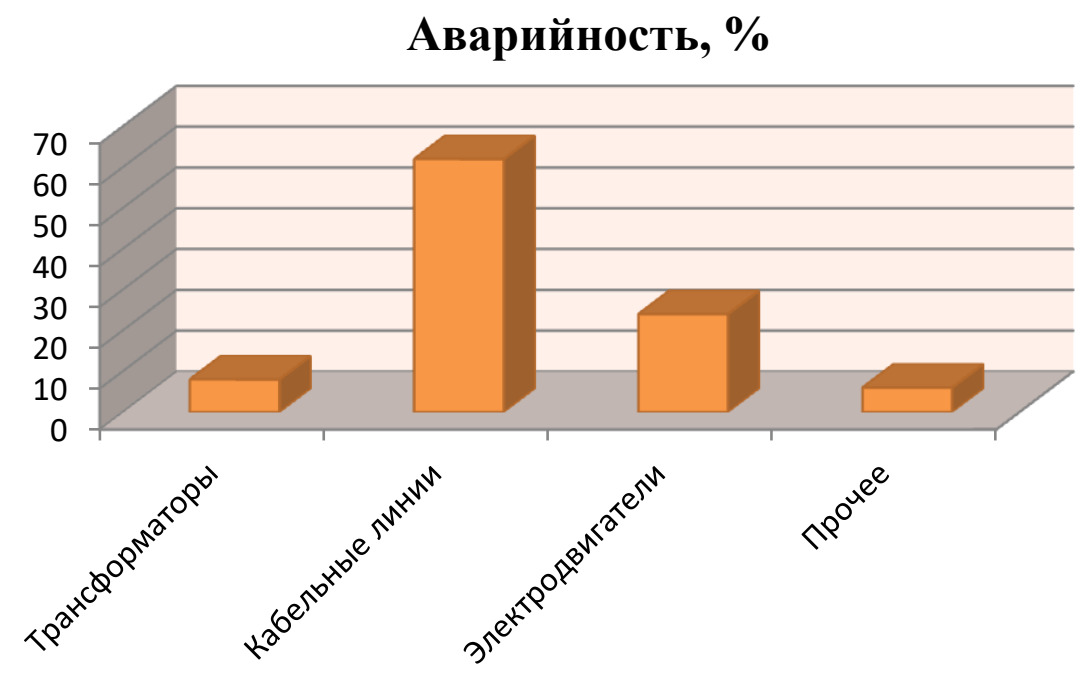

Рисунок 1. Аварийность электрооборудования нефтегазовой отрасли за период 2015-2018 гг.

Согласно статистическим сведениям, в большинстве случаев отказ кабелей происходит из-за пробоя изоляции во время испытаний повышенным выпрямленным напряжением (более 41 \%) (рисунок 2).

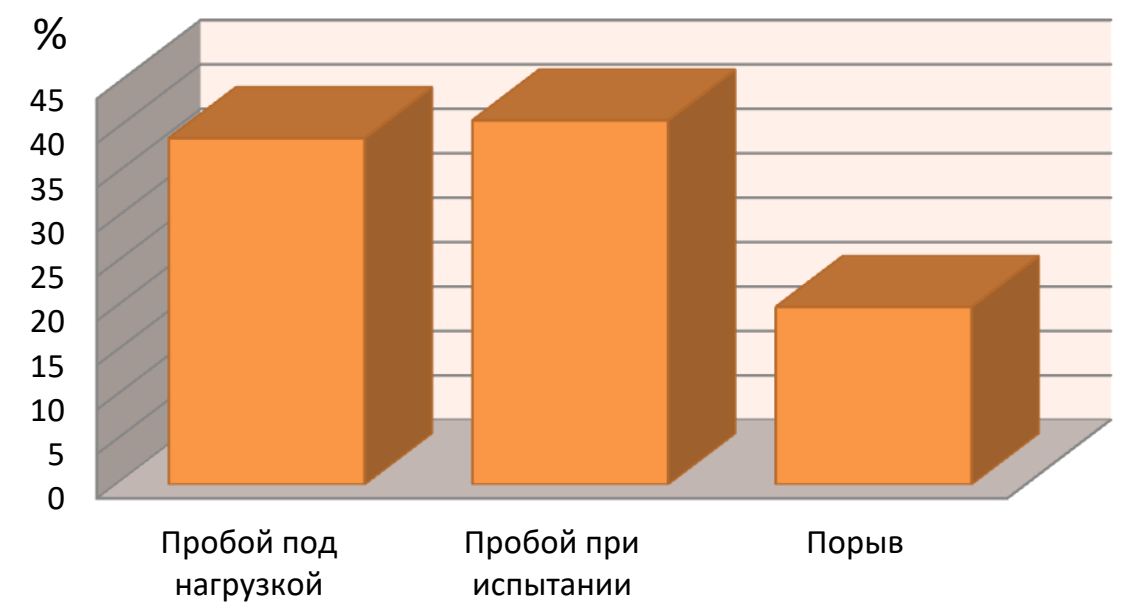

Рисунок 2. Причины выхода из строя кабельных линий

Данное испытание классифицируют как косвенно разрушающий метод, поэтому предлагается внедрение диагностики кабелей с использованием неразрушающего контроля, что позволит в скором времени отказаться от периодических испытаний разрушающими методами. В связи с этим был 
проведен анализ стадий развития нефтегазовой отрасли и интенсивность вложения в диагностику кабелей (рисунок 3). Анализ показал, что уровень «привлекательности» вложения в диагностику - средний. Это означает, что вложение в диагностику кабелей является целесообразным и поможет обеспечить и повысить энергоэффективность и безопасность на предприятиях нефтегазовой отрасли.

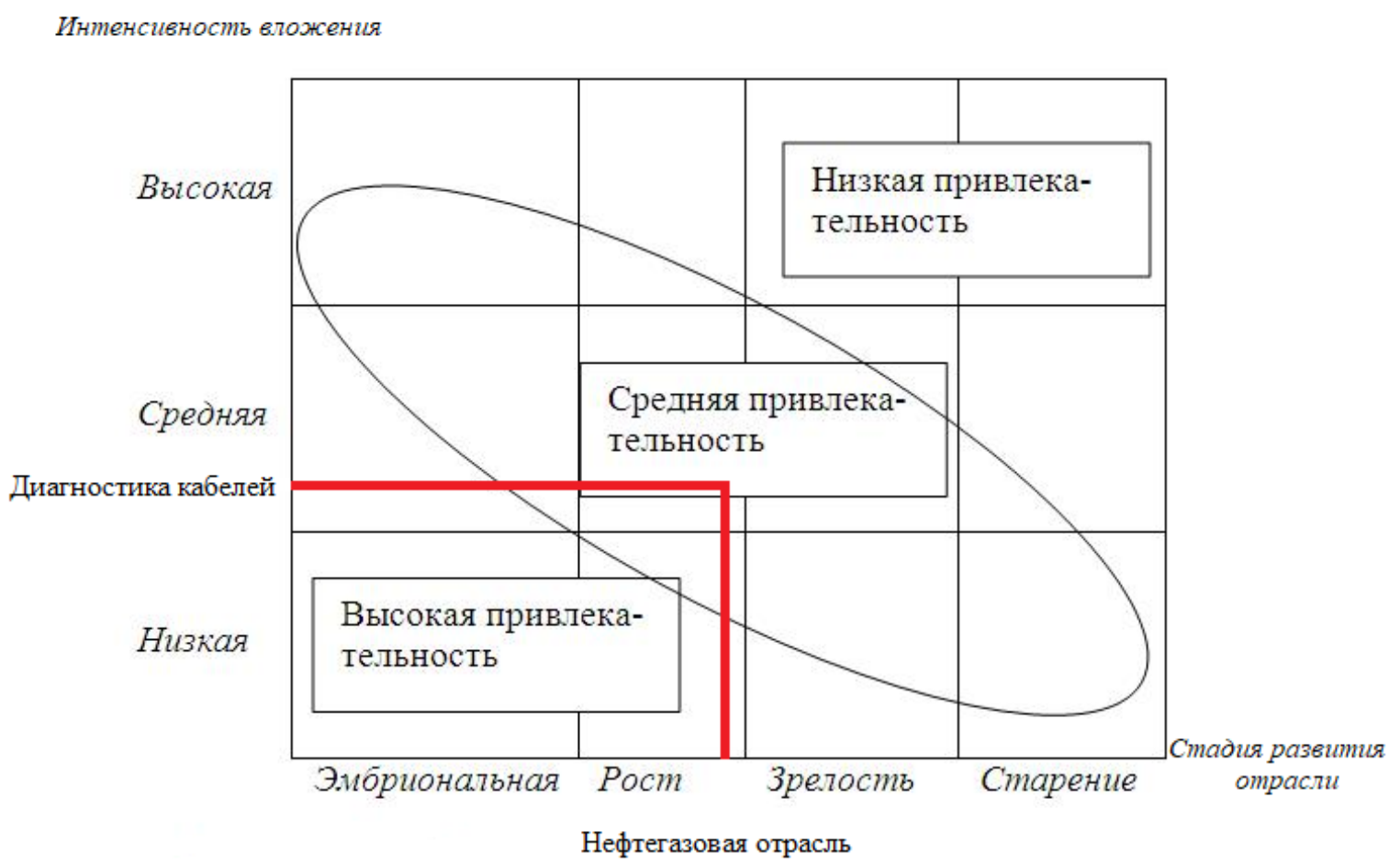

Рисунок 3. Уровень «привлекательности»

Диагностика кабеля обеспечит формирование единого подхода к предупреждению аварий и инцидентов, связанных с отказом кабельных линий, а также к повышению эффективности производства, зависящего от технического состояния кабеля.

Для объективной и полной оценки текущего технического состояния кабельных линий и для принятия управленческих решений предлагается метод количественной оценки, идентифицирующий повреждения и определяющий интегральный критерий.

Нахождение интегрального критерия позволит оценивать текущее техническое состояние и степень опасности кабеля [2]. 
Интегральный критерий формируется нейросетевым программным обеспечением из совокупности технических параметров кабеля, которые регламентированы такими правилами, как ПУЭ и ПТЭЭП, и диагностических параметров, полученных во время диагностирования кабеля, точнее, его рассмотрения как «черного ящика» с входными и выходными параметрами.

Данный интегральный критерий характеризует состояние кабеля и выдает результат о его техническом состоянии: нормальное или удовлетворительное, или неудовлетворительное.

Для реализации метода диагностики на основе данного критерия был составлен алгоритм, представленный на рисунке 4.

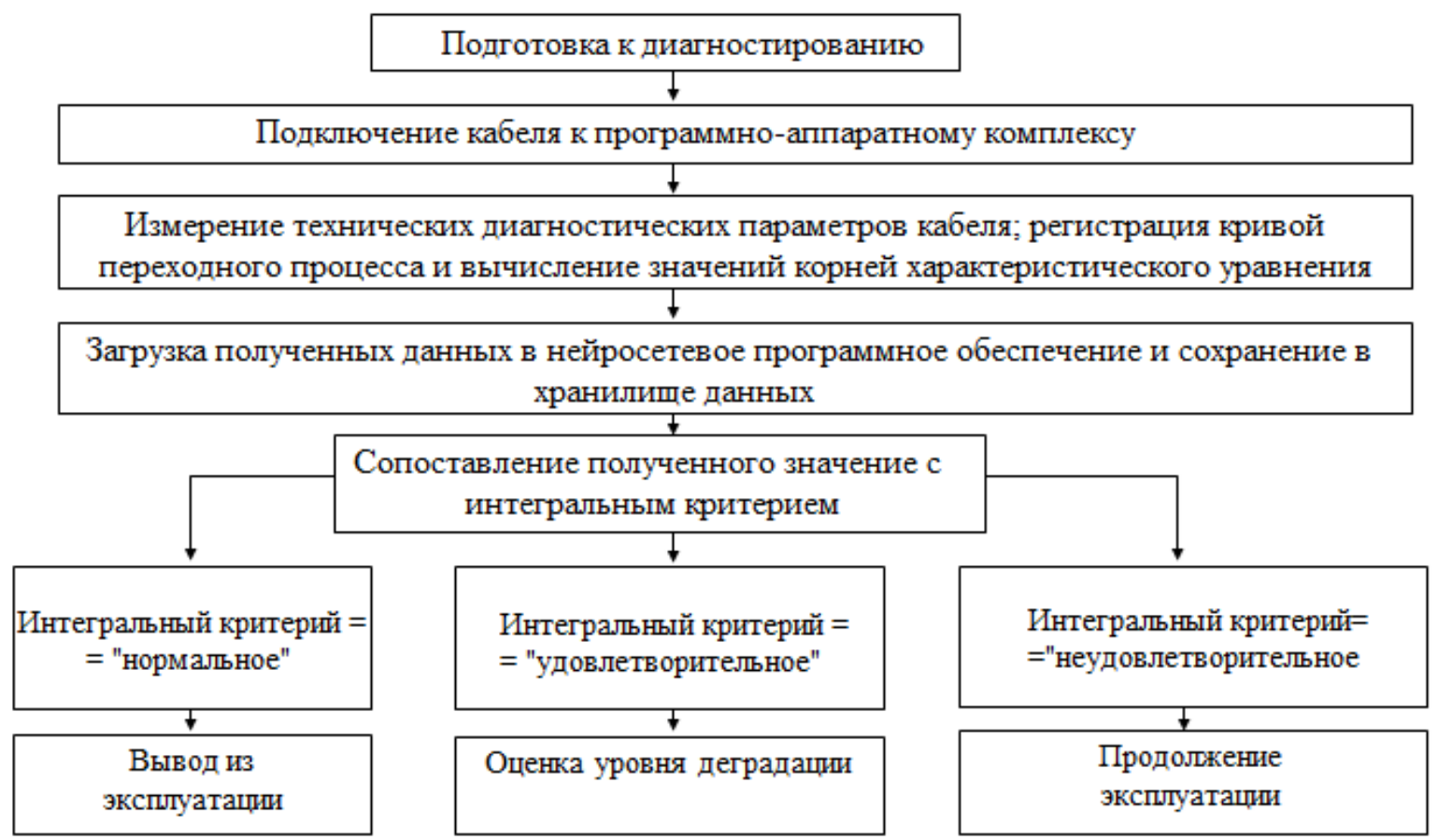

Рисунок 4. Алгоритм диагностики кабелей

Для любого внедрения нужны капитальные вложения. Затраты - это важный экономический показатель. Каждое вложение должно быть оправданным: эффективность и финансовую результативность вложений в диагностику кабелей устанавливают сравнением результата и затрат [3].

Экономической эффективностью при внедрении диагностики кабелей на предприятиях нефтегазовой отрасли считается снижение затрат и 
расходов, вызванных периодическими испытаниями и ремонтами кабельных линий.

Для расчета капитальных вложений и эксплуатационных расходов исходными данными являются рабочие характеристики сопоставляемых альтернатив диагностирования. В качестве основы возьмем имеющийся метод испытаний кабелей повышенным напряжением постоянного тока (таблица 1).

Таблица 1. Исходные данные для экономической оценки

\begin{tabular}{|l|c|c|}
\hline \multicolumn{1}{|c|}{ Показатели } & \multicolumn{1}{|l|}{$\begin{array}{c}\text { Испытание } \\
\text { повышенным } \\
\text { напряжением } \\
\text { постоянного тока }\end{array}$} & $\begin{array}{c}\text { Предлагаемый } \\
\text { нами метод } \\
\text { диагностики }\end{array}$ \\
\hline $\begin{array}{l}\text { Капитальные затраты (К), } \\
\text { тыс. руб. }\end{array}$ & 400 & 147,2 \\
\hline $\begin{array}{l}\text { Мощность энергопотребления, } \\
\text { кВт }\end{array}$ & 2,4 & 30 \\
\hline Время диагностирования, мин & 45 & 0 \\
\hline Уровень перебраковки, \% & 37 & 9,2 \\
\hline $\begin{array}{l}\text { Затраты на электроэнергию (3,), } \\
\text { тыс. руб. }\end{array}$ & 21,2 & \\
\hline
\end{tabular}

Для расчета срока окупаемости диагностики кабеля ежегодные амортизационные принимались как 5 \% от стоимости установки для диагностирования и учитывались расчетные стоимости кабеля [4]. Полученные технико-экономические расчеты занесли в таблицу 2.

Срок окупаемости равен 3,3 года.

Выполнена оценка риска от внедрения диагностики кабелей на предприятиях нефтегазовой отрасли, степень риска оценивается как средняя. 
Таблица 2. Технико-экономические расчеты

\begin{tabular}{|c|c|}
\hline Дополнительные текущие затраты (Э о), тыс. руб. & 45,3 \\
\hline Затраты на амортизацию ( $3_{\text {a }}$ ), тыс. руб. & 7,4 \\
\hline Затраты на обучение персонала ( $\left.3_{\text {об }}\right)$, тыс. руб. & 28,8 \\
\hline Суммарная экономия (Э), тыс. руб. & 4461,2 \\
\hline $\begin{array}{l}\text { Экономия от сокращения затрат, связанных с } \\
\text { уменьшением пробоя кабельной линии }\left(Э_{\text {к }}\right) \text {, тыс. руб. }\end{array}$ & 4440 \\
\hline Экономия на электроэнергию (Э э), тыс. руб. & 12,1 \\
\hline Суммарная годовая экономия (Э г), тыс. руб. & 4393,8 \\
\hline Срок окупаемости $\left(\mathrm{T}_{\mathrm{p}}\right)$, год & 3,3 \\
\hline
\end{tabular}

Риск: неожиданный скачок инфляции, который способен послужить причиной увеличения стоимости оборудования для диагностики, что, соответственно, повлечет повышение стоимости услуг.

Meры по сокращению риска: создание мероприятий по уменьшению себестоимости (нахождение альтернативы в плане оборудования) с целью вероятного регулирования её цены [5].

\section{Вывод}

Внедрение диагностики кабеля на предприятиях нефтегазовой отрасли будет эффективным и быстро окупится.

Использование диагностики позволит снизить затраты на замену кабеля при его выходе из строя по сравнению с применяемыми испытаниями за счет своевременной оценки его состояния и неразрушающего метода контроля. 


\section{Список используемых источников}

1. Абдрахманов Н.Х., Шайбаков Р.А. Совершенствование оценки рисков нефтеперерабатывающих предприятий // Безопасность труда в промышленности. 2007. № 12. С. 58-59.

2. Хафизов Ф.Ш., Новикова Фрейре Шавиер Ж. да К. Повышение эффективности эксплуатации кабельных линий с использованием комбинированной диагностики // Сетевое издание «Нефтегазовое дело». 2019. № 2. C. 6-15. URL: http://ogbus.ru/files/ogbus/issues/2_2019/ ogbus_2_2019_p6-15.pdf. (дата обращения: 06.07.19.)

3. Анисимов Н.К., Брейдо А.И. Организация, планирование и управление в хозяйстве сигнализации и связи. М.: Транспорт, 1979. $247 \mathrm{c}$.

4. Dugan R.C., McGranaghan M.F., Beaty H.W. Electrical Power Systems Quality. New York: McGraw-Hill, 1996. 265 p.

5. Thomson W.T., Fenger M. Current Signature Analysis to Detect Induction Motor Faults // IEEE Industry Application Magazine. 2001. Vol. 7. No. 4. P. 26-34. DOI: 10.1109/2943.930988.

\section{References}

1. Abdrakhmanov N.X., Shaibakov R.A. Sovershenstvovanie otsenki riskov neftepererabatyvayushchikh predpriyatii [Improving Risk Assessment of Oil Refineries]. Bezopasnost' truda v promyshlennosti - Occupational Safety in Industry, 2007, No. 12, pp. 58-59. [in Russian].

2. Khafizov F.Sh., Novikova Freire Shavier Zh. da K. Povyshenie effektivnosti ekspluatatsii kabel'nykh linii $\mathrm{s}$ ispol'zovaniem kombinirovannoi diagnostiki [Improving the Efficiency of Operating Cable Lines Using Combined Diagnostics]. Setevoe izdanie «Neftegazovoe delo» - Online Edition «Oil and Gas Business», 2019, No. 2, pp. 6-15. URL: http://ogbus.ru/files/ ogbus/issues/2_2019/ogbus_2_2019_p6-15.pdf. (accessed 06.07.19.). [in Russian]. 
3. Anisimov N.K., Breido A.I. Organizatsiya, planirovanie $i$ upravlenie $v$ khozyaistve signalizatsii i svyazi [Organization, Planning and Management of Alarm and Communication Facilities]. Moscow, Transport Publ, 1979. 247 p. [in Russian].

4. Dugan R.C., McGranaghan M.F., Beaty H.W. Electrical Power Systems Quality. New York, McGraw-Hill, 1996. 265 p.

5. Thomson W.T., Fenger M. Current Signature Analysis to Detect Induction Motor Faults. IEEE Industry Application Magazine, 2001, Vol. 7, No. 4, pp. 26-34. DOI: 10.1109/2943.930988.

\section{Сведения об авторе}

\section{About the author}

Новикова Фрейре Шавиер Ж. да К., аспирант кафедры «Пожарная и промышленная безопасность», УГНТУ, г. Уфа, Российская Федерация

G. da K. Novikova Freyre Shavier, Post-graduate Student of Fire and Industrial Safety Department, USPTU, Ufa, Russian Federation e-mail: shavier_93@mail.ru 